February 27, 2002

LBNL-48917-REV

\title{
The Basis Problem in Many-Worlds Theories
}

\author{
Henry P. Stapp \\ Lawrence Berkeley National Laboratory \\ University of California \\ Berkeley, California 94720
}

\begin{abstract}
It is emphasized that a many-worlds interpretation of quantum theory exists only to the extent that the associated basis problem is solved. The core basis problem is that the robust enduring states specified by environmental decoherence effects are essentially Gaussian wave packets that form continua of non-orthogonal states. Hence they are not a discrete set of orthogonal basis states to which finite probabilities can be assigned by the usual rules. The natural way to get an orthogonal basis without going outside the Schroedinger dynamics is to use the eigenstates of the reduced density matrix, and this idea is the basis of some recent attempts by many-worlds proponents to solve the basis problem. But these eigenstates do not enjoy the locality and quasi-classicality properties of the states defined by environmental decoherence effects, and hence are not satisfactory preferred basis states. This core problem needs to be addressed and resolved before a many-worlds-type interpretation can be said to exist.
\end{abstract}

${ }^{*}$ This work is supported in part by the Director, Office of Science, Office of High Energy and Nuclear Physics, Division of High Energy Physics, of the U.S. Department of Energy under Contract DE-AC03-76SF00098 


\section{Introduction.}

The basis problem of the many-worlds interpretation can be efficiently introduced by quoting a few exerpts from the entry "Everett's Relative-State Formulation of Quantum Mechanics" in the Stanford Encyclopedia of Philosophy. That entry is available online [1], and elaborates on the points summarized in the following passages:

Everett's relative-state formulation of quantum mechanics is an attempt to solve the measurement problem by dropping the collapse dynamics from the standard von Neumann-Dirac theory of quantum mechanics. The main problem with Everett's theory is that it is not at all clear how it is supposed to work. In particular, while it is clear that he wanted to explain why we get determinate measurement results in the context of his theory, it is unclear how he intended to do this. There have been many attempts to reconstruct Everett's no-collapse theory in order to account for the apparent determinateness of measurement outcomes. These attempts have led to such formulations of quantum mechanics as the many-worlds, many-minds, many-histories, and relative-fact theories. Each of these captures part of what Everett claimed for his theory, but each also encounters problems.

...

Everett's no-collapse formulation of quantum mechanics was a reaction to problems that arise in the standard von NeumannDirac collapse theory. Everett's proposal was to drop the collapse postulate from the standard theory, then deduce the empirical predictions of the standard theory as the subjective experiences of observers who are themselves treated as physical systems described by his theory. It is, however, unclear precisely how Everett intended for this to work. Consequently, there have been many, mutually incompatible, attempts at trying to explain what 
he in fact had in mind.

$\cdots$

This is what Everett says: "We shall be able to introduce into [the relative-state theory] systems which represent observers. Such systems can be conceived as automatically functioning machines (servomechanisms) possessing recording devices (memory) and which are capable of responding to their environment. The behavior of these observers shall always be treated within the framework of wave mechanics. Furthermore, we shall deduce the probabilistic assertions of Process 1 [rule 4b] as subjective appearances to such observers, thus placing the theory in correspondence with experience. We are then led to the novel situation in which the formal theory is objectively continuous and causal, while subjectively discontinuous and probabilistic. While this point of view thus shall ultimately justify our use of the statistical assertions of the orthodox view, it enables us to do so in a logically consistent manner, allowing for the existence of other observers."

Everett's goal then was to show that the memory records of an observer as described by his no-collapse theory would match those predicted by the standard theory with the collapse dynamics. The main problem in understanding what Everett had in mind is in figuring out how this correspondence between the predictions of the two theories was supposed to work.

..

\section{Many Worlds}

DeWitt's many-worlds interpretation is easily the most popular reading of Everett. On this theory there is one world corresponding to each term in the expansion of $\mathbf{E}$ when written in the preferred basis (there are always many ways one might write the quantum-mechanical state of a system as the sum of vectors in 
the Hilbert space; in choosing a preferred basis, one chooses a single set of vectors that can be used to represent a state and thus one chooses a single preferred way of representing a state as the sum of vectors in the Hilbert space). The theory's preferred basis is chosen so that each term in the expansion of $\mathbf{E}$ describes a world where there is a determinate measurement record. Given the preferred basis (surreptitiously) chosen above, $\mathbf{E}$ describes two worlds: one where $J$ (or perhaps better $J 1$ ) determinately records the measurement result "spin up" and another where $J$ (or J2) determinately records "spin down".

DeWitt and Graham describe their reading of Everett as follows: "[Everett's interpretation of quantum mechanics] denies the existence of a separate classical realm and asserts that it makes sense to talk about a state vector for the whole universe. This state vector never collapses and hence reality as a whole is rigorously deterministic. This reality, which is described jointly by the dynamical variables and the state vector, is not the reality we customarily think of, but is a reality composed of many worlds. By virtue of the temporal development of the dynamical variables the state vector decomposes naturally into orthogonal vectors, reflecting a continual splitting of the universe into a multitude of mutually unobservable but equally real worlds, in each of which every good measurement has yielded a definite result and in most of which the familiar statistical quantum laws hold."

- .

Perhaps more serious, in order to explain our determinate measurement records, the theory requires one to choose a preferred basis so that observers have determinate records (or better, determinate experiences) in each term of the quantum-mechanical state as expressed in this basis. 
These quotes from Barrett's 1998 article in the Stanford Encyclopedia of Philosophy emphasize the central importance of specifying a basis, and it gives many references to fill in and back up the description it gives of the contemporary situation. However, that review does not mention David Deutsch's ambitious and detailed 1985 attempt[2] to solve the basis problem. That omission is justified by the fact that Deutsch's approach was found by Sara Foster and Harvey Brown[3] to be marred by many logical flaws. But in spite of these flaws, it will be useful to describe Deutsch's 1985 attempt in order to get an idea of the problems involved in the construction of a many-worlds interpretation.

\section{Deutsch's 1985 Proposed Solution of the Many-Worlds Basis} Problem.

Deutsch clearly recognized that a key problem in creating a many-worlds interpretation is the problem of defining the preferred basis. He calls it the 'interpretation basis', and his forty-page paper is devoted largely to task of defining it. The other main problem is to understand how a relative probabilility comes to be associated with each of the alternative possibilities, all of which occur jointly and concurrently in the evolving quantum state. Since the occurrence of any one of the possible outcomes is accompanied by the occurrence of each of the other possible outcomes it is a problem to understand, solely on the basis of the Schroedinger equation alone, how one of the possibilities can be, say, a billion times less likely to appear than some other one. I shall not delve here into this interesting problem, but will focus on the basis problem.

Solving the basis problem seems to demand mathematically detailed rules that specify (1), when the full reality splits into these distinct worlds, or branches, (2), what these separate parts are, and (3), which variables associated with these different worlds, or branches, acquire, or possess, definite values in each such world, or branch. Deutsch recognized the three aspects of the problem, and tried to provide well defined answers to each.

Of course, every interpretation of quantum theory that goes beyond the 
pragmatic Copenhagen approach of merely providing a set of rules the connect human experiences must provide analogous kinds of rules. But the mathematical requirements on the structure of these rules is far more stringent for a many-world theory because such a theory allows no collapse, and everything must be determined exclusively in terms of the one basic reality, which is the one quantum state of the universe that evolves in accordance with the Schroedinger equation: no other process is allowed. But as Foster and Brown emphasize, in collapse theories the specification of these rules "need only be within the context of some theory, not necessarily quantum theory." The encompassing theory could allow, for example, as von Neumann's theory does, experiential realities to enter dynamically into the determination of the basis, instead of being mere consequences of some choice of basis that is itself wholly determined by the unitary evolution of the quantum state in accordance with the Schroedinger equation.

Deutsch recognized and stressed the critical importance of this basis problem, and made a determined effort to solved it:

"The remedy which I propose in section 7 is that an extra rule be appended to the quantum formalism, specifying how the interpretational basis depends at each instant of time on the physical state and dynamical evolution of a quantum system."

The first problem that he tries to resolve is what he calls "the notorious difficulty of specifying the preferred instants $t \ldots$ when a measurement is completed." These instants must be defined solely in terms of (1), the single evolving state of the universe, which evolves always via the Schroedinger equation, and (2), the law that governs this continuous unitary evolution.

The second problem is that at each of these distinct "instants of splitting" the unified actual universe must be decomposed in a specified way into two systems, the 'observed system' and 'observing system'. Hence the rule that defines how the unified universe is to be split at that instant into these two special parts must be specified in terms solely of the state vector itself and its law of evolution. 
The third problem is to define for each instant a set of evolving basis vectors so that the state of the universe can at every instant be decomposed in a unique way into a sum of component vectors that can be associated with different worlds, or branches of reality, that possess definite values for the quantities "measured" at each instant of splitting.

Deutsch solves the problem of dividing the unified physical world into the well defined physical parts that will ultimately acquire 'possessed values' by restricting his considerations to a model world that has only a finite set of states, and dividing this set of states in some way into subsystems each having a 'prime number' of states in each subsystem. One evident problem with that approach is the essential role played by this finiteness assumption and the 'prime factor' rule: Deutsch does not indicate how this 'prime number rule', which is purely kinematic (it does not involve the equations of motion) will carry over to real systems, in which every particle carries with it an infinite number of states, and in particular to the systems that carry the stored memories associated with our experiences. These systems are presumably made of many particles, and are strongly dependent on the dynamics.

Once the separation of the universe into the observed and observing systems is made, Deutsch specifies the instant at which the splitting occurs to be essentially the instant at which the interaction between observed and observing system vanishes. But the ubiquitous tails of real systems would make that condition difficult to satisfy in the physical universe.

Once the instants of splittings are fixed, and the separation of the universe at each of these instants into the observed and observing system is specified, one needs to define, for every instant of time, the preferred basis. This basis defines the decomposition of the quantum state of the universe into a unique sum of vectors each of which is parallel to one of the vectors in the preferred set of basis vectors. Each of these particular component vectors is supposed to correspond to a separate world, or branch of the universe. The decomposition must be such that if a probability is assigned to each such world in accordance with usual rule, which equates it to the square of 
the length of the corresponding vector, then the expectation values of certain special quantities, namely those represented by operators whose eigenvectors are these basis vectors, are required to agree with the general quantum mechanical rule for expectation values. This is how Deutsch proposed to endow the evolving non-collapsing quantum universe with some definite outcomes in a way concordant with the quantum probability rules: each individual branch would have 'possessed values' appropriate to that branch, and the branch would be given the appropriate statistical weight. But the entire construction depends crucially on the idea that a particular well-defined set of preferred basis states is specified by the evolving non-collapsing quantum state of the universe.

Deutsch tried to deduce the structure of this preferred basis set by imposing the requirement of no-faster-than-light-influence, but his argument is, as was correctly noted by Foster and Brown, logically flawed. The primary condition on a many-world interpretation is that there be no actual collapse: the values associated with the preferred basis vectors are supposed to be "possessed" by the systems, or to exist within nature, without there being any collapse that actually eliminates the cross terms. However, the crucial equation (Eq. 50) that gives the structure of the preferred basis does not follow from Deutsch's argument, insofar as the no collapse condition is maintained. The result would follow if the appropriate collapse were to occur. That is, Deutsch's proof would be valid if he were trying to define the preferred basis in the context of a collapse-type theory, but it is not valid in the many-worlds context. This error is not just a small slip-up that can by fixed by a minor correction. Foster and Brown emphasize this problem, and also problems with the existence and uniqueness of solutions to the conditions that Deutsch proposes. Michael Lockwood reports in his 1989 book Mind, Brain, and the Quantum, (p. 236) that Deutsch "has failed so far to find any plausible way to meet Foster and Brown's objections." Lockwood's own solution brings in "Mind" in an important way, and is thus not a "Many-Worlds Interpretation" in the DeWitt-Deutsch sense of this term. 


\section{The Core Basis Problem}

This section identifies the core basis problem.

The essential point is that if the universe has been evolving since the big bang in accordance with the Schroedinger equation, then it must by now be an amorphous structure in which every device is a smeared-out cloud of a continuum of different possibilities. Indeed, the planet earth would not have a well defined location, nor would the rivers and oceans, nor the cities built on their banks. Due to the uncertainty principle, each particle would have had a tendency to spread out. Thus various particles with various momenta would have been able to combine and condense in myriads of ways into bound structures, including measuring devices, whose centers, orientations, and fine details would necessarily be smeared out over continua of possibilities. The quantum state would be, to first order, a superposition of a continuum of slightly differing classical-type worlds with, in particular, each measuring device, and also each observing brain, smeared out over a continuum of locations, orientations, and detailed structures. But the normal rules for extracting well defined probabilities from a quantum state require the specification, or singling out, of a discrete set (i.e., a denumerable set) of orthogonal subspaces, one for each of a set of alternative possible experientially distinguishable observations. But how can a particular discrete set of orthogonal subspaces be picked out from an amorphous continuum by the action of the Schroedinger equation alone?

For example, if one has in the wave function of the universe, in addition to the contribution from some particular wave function of a Stern-Gerlach device, also contributions from all slight deviations of this wave functions from this original form, then this collection, if no one deviation is singled out from its immediate neighbors in some way, defines no proper subspace: any collection of vectors in a full neighborhood of a vector spans the entire vector space. This fact poses a problem in principle for any deduction of probabilities from the Schroedinger dynamics alone: how can a specific set discrete orthonormal subspaces be specified by the continuous action of the 
Schroedinger equation on a continuously smeared out amorphous state?

The essential role of the human participant/observer in the orthodox Copenhagen interpretation is to pick out a particular discrete subspace (or a particular set of orthogonal subspaces) from a continuum of logically possible alternatives. He is able to do this by not being part of the universe that evolves via the Schroedinger equation. The fact that the participant/observer who chooses the experiment, and observes the outcome, stands, in this sense, outside the quantum universe, but acts upon it and observes it, is a key logical aspect of the orthodox interpretation. In the von Neumann version this choice is achieved by the notorious "process I", which likewise is not determined by the unitary Schroedinger process, but specifies a particular way of dividing the full Hilbert space into a denumerable set of orthogonal subspaces.

Any theory that proposes that the entire universe be controlled by the Schroedinger equation alone must explain how that continuous unitary process can pick out a particular denumerable set of 'observable' orthogonal subspaces with nonzero probabilities, in such a way that these nonzero probabilities become associated with empirically experienced alternatives.

Most contemporary efforts to solve the "measurement" problem in quantum theory rest heavily upon "environmental decoherence effects." One of the effects of this interaction of a system with its environment is to convert what would otherwise be a wave packet that can extend coherently over a large distance, in various dimensions, into what is essentially a mixture of narrow gaussian wave packets. The mixture is, essentially, a sum over a continuum of displaced narrow Gaussians in the variable associated with the wave function [4]. These Gaussian states are dynamically robust under the effects of interactions with the environment, and have classical-type properties that make then good candidates for the states that correspond to definite experiences.

Specifically, the effect of environmental decoherence is to reduce a typical 
state $\Psi\left(x^{\prime}\right)$ to a mixture of states of the form

$$
\Psi_{x}\left(x^{\prime}\right)=N(x) \exp -a^{2}\left(x^{\prime}-x\right)^{2},
$$

where $N(x)$ is a weight factor, and the subscript $x$, which labels the centerpoint, or peak, of the Gaussian wave packet, ranges over a continuum of values. None of these states is orthogonal to any other. (Here, to make the idea clear, I am exhibiting only one single continuum variable, $x$. But all position-type variables would be affected in a similar way.)

This decoherence mechanism eliminates certain interference effects, but it does not solve the basis problem. There will be a continuum of these Gaussians, (3.1), and they overlap: i.e., they are not orthogonal. As before, if one takes a set of these state consisting of one of them together with those displaced from it by a distance $\Delta x$ less than any epsilon greater than zero then this set will included a continuum of states that span a large part of the Hilbert space of functions of the variable $x^{\prime}$ (See [5]). Since no one of this continuum of states is singled out from the rest by the Schroedinger dynamics, and they jointly span a large part of the Hilbert space in question, one does not immediately obtain from the Schroedinger dynamics plus decoherence the needed denumerable set $\left\{P_{i}\right\}$ of orthogonal projection operators, or the denumerable set of orthogonal subspaces that they specify.

A natural candidate for the needed denumerable set of orthogonal subspaces associated with the continuum of states (3.1) is the set of subspaces defined by the different eigenvalues of the reduced density matrix associated with that mixture of states. That candidate is, in fact, the one chosen by Deutsch in his 1985 paper. However, that choice is not satifactory. One wants states that are highly localized, like a classical state. But the eigenstates of the reduced density matrix associated with the states (3.1), i.e., the eigenstates of a density matrix of the form

$$
<x^{\prime}\left|\rho_{r}\right| x^{\prime \prime}>=N\left(x^{\prime}, x^{\prime \prime}\right) \exp -a^{2}\left(x^{\prime}-x^{\prime \prime}\right)^{2} / 2,
$$

with a slowly changing normalization function $N\left(x^{\prime}, x^{\prime \prime}\right)$, are spread out over 
large distances, rather than being compressed into small regions, like the states (3.1) themselves are. Taking the eigenstates of the reduced density matrix goes in the wrong direction. Because this natural choice is unsatisfactory one is left with the core problem of how to specify, without appeal to any process except the continuous Schroedinger unitary evolution, the denumerable set of orthogonal subspaces that are to be associated with definite observed values and finite probabilities.

\section{Deutsch's Multiverse Proposal}

Recently Deutsch has put forward a new many-worlds concept labelled the "multiverse". In the popular account given in his book The Fabric of Reality the multiverse would appear to be a collection of classical-type universes each one similar to the single quasi-classical world introduced by David Bohm in his famous pilot-wave model. But that cannot be Deutsch's real meaning because Bohm's classical-type universe is governed, in part, by nonlocal forces, and a key virtue of the many-worlds approach, often emphasized by Deutsch, is that it does not involve or entail any kind of faster-than-light transfer of information.

In his recent paper The Structure of the Multiverse [6] Deutsch gives a more detailed glimpse into his thinking, and he emphasizes at the outset the existence of the basis problem, and the demand for no faster-than-light transfer of information. Deutsch's new proposal involves different quasi-classical aspects of the quantum universe that persist and evolve in parallel over finite temporal intervals but can eventually recombine to produce quantum interference effects. This idea is much closer to standard ideas than Bohm's model, in which the quasi-classical world persists for all time, and quantum interference effects are explained by a special nonlocal force, rather than by the merging of previously distinct quasi-classical components.

In his new work Deutsch again emphasizes the basis problem, and I think it is fair to say that a many-worlds interpretation is not defined until it solves in principle the basis problem. Indeed, the basis problem is the problem that any interpretation of quantum theory must resolve in some way. Thus the 
central idea of the Copenhagen interpretation was to imbed the quantum system in a larger system that specifies the preferred basis by bringing in "measuring devices" that are set in place by a classically conceived process. In von Neumann's formulation there is the infamous "Process I", which likewise lies outside the process governed by the Schroedinger equation. The key challenge that must be met by a satisfactory many-worlds interpretation is to solve this basis problem within quantum theory itself, without bringing in any outside dynamical process.

Deutsch's program is to create a satisfactory many-worlds-type theory by considering nature to be a quantum computer. Deutsch cites Feynman's paper[]] on quantum mechanical computers. In this paper Feynman says:

Of course, there are external things involved in making the measurements and determining all this [setting up the computer], which are not parts of our computer. Surely a computer has eventually to be in interaction with the external world, both for putting in data and for taking it out.

Here Feynman is following the orthodox idea that a computer is a quantum system, and as such must be considered imbedded in a larger world in which we prepare initial conditions, including the structures of all devices, and then extract outcomes. Deutsch's problem is, accordingly, to show how by considering the whole universe to be a quantum computer one can solve this heretofore unresolved interpretational problem that has beset those trying to create a satisfactory many-worlds theory.

To emphasize the problems that others have encountered I quote some passsages from Zurek's 1998 review 8 of the status of attempts to use environmental decoherence (abetted, if helpful, by coherent histories) to solve the interpretational problem.

It will be emphasized that while significant progress has been made ... much remains to be done on several fronts which all 
have implications on the overarching question of interpretation. $\cdots$

Thus, while what follows is perhaps the most complete discussion of the interpretation implied by decoherence, it is still only a report of partial progress.

...

The advantages of Everett's original vision was to reinstate quantum theory as a key tool in search of its own interpretation. The disadvantages (which were realized only some years later, after the original proposal became more widely known) include (i) the ambiguity in what constitutes the branches ... (ii) re-emergence of the questions about the origin of probabilities ... (iii) it was never clear how to reconcile unique experiences of observers with the multitude of alternatives present in the MWI wave function. ...

The interpretation based on ideas of decoherence and [environmentally induced] einselection has not really been spelled out to date in any detail. I have made a few half-hearted attempts in this direction, but, frankly, I was hoping to postpone this task, since the ultimate questions tend to involve such 'anthropic' attributes of the 'observership' as 'perception', 'awareness' or 'consciousness',

...

Einselection chooses a preferred basis ... İt will often turn out that it is overcomplete. Its states may not be orthogonal, and, hence, they could never follow from the diagonalization of the density matrix.

These quotations show that Zurek recognizes the core continuum problem that I have been describing.

How then does Deutsch deal with this basic problem, which is that a 
quantum universe evolving via the Schroedinger equation does not seem to be able to pick out a discrete set of preferred directions (or subspaces) in Hilbert space that specify quasi-classical properties that can be assigned definite "possessed values" with well defined probabilities.

His approach is first to define, in terms of his quantum-computer formulation of quantum theory, certain mathematical requirements for a portion of the full quantum computer to be 'behaving classically'. These requirements depend upon certain directions in Hilbert space being singled out as the ones that correspond to 'possessed values'. Those special directions are not specified beforehand, but are defined by the dynamics, in the sense that if with some choice of these directions a certain system behaves classically then these special directions are picked out by that classicality condition itself. Thus the preferred basis is defined dynamically, by a classicality condition.

This is eminently reasonable, as far as it goes. By it does not address the basic continuum problem, which would be to show that the classicality conditions specify, at each moment at which "possessed values" are defined, a denumerable set of mutually orthogonal subspaces within the full amorphous many-worlds universe, in which there will be continua of neighboring environmentally robust configurations in which the detectors that define the preferred axes of his qubit-measuring devices are slightly re-oriented. The basic problem is to get out of the continua specified by the Schroedinger dynamics plus environmental decoherence the denumerable sets of orthogonal subspaces needed to make the probability formulas work. This core issue was not addressed.

\section{Zurek's Approach and Records}

Deutsch's multiverse theory is in general accord with Zurek's approach [8], and in the end Deutsch appeals to Zurek's arguments to support the idea that the 'classically behaving' aspects of the quantum universe will explain the 'classical world' in which we live and act.

Zurek's treatment is wrapped up, as it should be, with his treatment of probabilities. In this connection he says: 
A true 'frequency' with a classical interpretation cannot be defined at a level which does not allow 'events' - quantum evolution which leads to objectively existing states - to be associated with the individual members of that ensemble. ... The reduced density matrix $\rho$, which emerges following the interaction with the environment, and a partial trace will always be diagonal in the same basis of einselected pointer states $\{\mid i>\}$. These states help define elementary 'events'. Probabilities of such events can be inferred from their coefficients in $\rho$, which have the desired 'Born rule' form.

Zurek's discussions of 'existing' values and probabilities rests on this model, in which the reduced density matrix is diagonal in the same states that specify the values that are supposed to 'exist'. But that model must come to grips with the continuum problem, which is emphasized elsewhere in his paper by statements such as:

In that case [which in fact arises in the quantum field theoretic treatment of the measurement process] the states which are most immune to decoherence in the long run turn out to be ... Gaussians. ... Hence coherent states [which are Gaussians] are favored by decoherence. ... Einselection chooses a preferred basis in the Hilbert space in recognition of its predictability. That basis will be determined by the dynamics of the open system in the presence of environmental monitoring. It will often turn out that it is over complete. [This is precisely the case for the Gaussian's mentioned above.] Its states may not be orthogonal, and, hence, they would never follow from diagonalization of the density matrix.

Thus Zurek stresses that the robust Gaussian-type states picked out by environmental decoherence belong to a continuum of non-orthogonal states, and hence do not generally define a denumerable set of orthogonal subspaces, 
and are not the basis states defined by diagonalization of the reduced density matrix. This leads to the question: Does the diagonalization of the reduced density matrix yield physically appropriate basis states?

A partial answer this question is obtained by noting that for constant $\rho$ the reduced density matrix is essentially

$$
\begin{gathered}
<x^{\prime}\left|\rho_{r}\right| x^{\prime \prime}>=\sqrt{2 / \pi} \int d x \exp -\left(x^{\prime}-x\right)^{2}<x^{\prime}|\rho| x^{\prime \prime}>\exp -\left(x^{\prime \prime}-x\right)^{2} \\
=\exp -(1 / 2)\left(x^{\prime}-x^{\prime \prime}\right)^{2}<x^{\prime}|\rho| x^{\prime \prime}>
\end{gathered}
$$

The eigenfunctions of this $\rho_{r}$ are the functions exp -ipx, which are spread uniformly over all space. (To get normalized functions one can formulate the problem with periodicity over a large interval.) Thus in this simplest case the diagonalization effectively undoes the localization effect produced by the decoherence: it does not produce localized, robust, quasi-classical states.

Zurek emphasizes, as did Everett, the critical importance of records. A record is typically stored in the state of a relative coordinate in which the potential energy has two deep, well separated wells. It has been suggested, in this case, that if the energy gap between, on the one hand, the two almost degenerate states of lowest energy and, on the other hand, the higher-energy double-well energy eigenstates is large compared to the energy of the pertinent environmental states then those higher-energy states can be ignored. The problem then reduces to one in which only the two lowest energy states are considered, together with the states of the environment. The reduced density matrix would be a $2 \times 2$ matrix that, apart from an overall normalization factor, has $(1+b, 1-b)$ on the diagonal and the value $a$ in the two off diagonal slots, with $a$ generally much smaller than $b$. The two eigenstates of this reduced density matrix will, up to corrections of order $a / b$, each be concentrated in one well or the other, which is the desired result.

This approximation is essentially equivalent to concentrating the coordinate space wave function at two points, and neglecting the connecting network of coordinate-space points. However, there is a qualitative difference between setting the other elements to zero and taking them to be very tiny. 
To illustrate this point consider first the easily solvable case of the $3 \times 3$ matrix with diagonal elements $(1, c, 1)$, next-to-diagonal elements $a>0$, and zeros in the two corners. The eigenvalues are $(1,(1+c+r) / 2,(1+c-r) / 2)$, with $r=\sqrt{ }\left((1-c)^{2}+8 a^{2}\right)$, and the eigenvectors are $(1,0,-1),(1, s+, 1)$ and $(1, s-, 1)$, with $s \pm=4 a /(1-c \pm r)$. For $a$ and $c$ very small, but nonzero, one has $s+\approx 2 a$, and $s-\approx-1 / a$, and hence the triad of eigenstates is unlike the triad of localized states

$$
(1,0,0),(0,1,0),(0,0,1)
$$

which are possible eigenstates for $a=0$.

This example illustrates the general fact that for almost any matrix that is symmetric along the diagonal [i.e., with $\langle i|M| j\rangle=<-i|M|-j\rangle$, with the states labeled $(n, n-1, \ldots,-n)]$, and that has $\langle i|M| j>\neq 0$ for all pairs $(i . j)$, all of the eigenstatates will be either symmetrical or anti-symmetrical: this property of our $3 \times 3$ example carries over to the general symmetric (along the diagonal direction) case. [One can prove this result by making the anzatz that the eigenstates are either symmetric or antisymmetric [i.e., $<i|\phi>= \pm<-i| \phi>$ ] and noting that the equations for the symmetric and antisymmetric eigenvectors have $n+1$ and $n$ solutions, respectively, and that equations for the symmetric and antisymmetric eigenfunctions are different.]

This result for the exactly symmetrical case carries over to the nearby almost-symmetrical cases by analyticity. But the general rule for specifying the preferred basis must cover the special case of the nearly symmetrical potential wells. Since the diagonalization of the reduced density matrix does not lead to the correct (i.e., localized) eigenstates in the nearly symmetrical cases it cannot be the correct general rule. Thus although the diagonalization of the reduced density matrix does define a denumerable set of orthogonal subspaces, it cannot always do the job that the participant/observer does in orthodox Copenhagen quantum theory, and that "process I" does in von Neumann quantum theory, which is to pick out a discrete set of orthogonal subspaces that are associable with alternative possible experiences. 
The example of the nearly symmetrical case shows that the diagonalization of a reduced density matrix does not define the needed discrete orthogonal states. Robustness is the essential quality. More specifically, it is the quality of a "record" not only to endure, but to be creatable, reproducible, and able to control essentially classical behaviors without being appreciably degraded. [9] The effects of the environment are important in this connection, because they destroy the possibility that certain states could be records. Thus environmental decoherence does have powerful effects. But that does not solve the problem of extracting, by means of the Schroedinger equation alone - from the amorphous state that is created by the evolution of the big bang state via the Schroedinger equation - a discrete set of orthogonal basis record-type states. In this Schroedinger state every atom, and conglomeration of atoms, will be a smeared out continuum. The essential reason why the founders had to regard the quantum system as being imbedded in an enveloping process, and why von Neumann had to introduce his Process I, was to solve this problem of specifying a discrete basis for the probability computations associated with our apparently discrete (Yes or No) experiences, when the Schroedinger equation generates continuous evolution of an ever-amorphous state. Specifing a discrete set of basis states solely from the continuous structure generated by the Schroedinger equation would appear to be an impossible feat. In any case, no claim to having constructed a rationally coherent many worlds/minds formulation of quantum theory is justified unless this discrete basis problem is clearly recognized and adequately resolved. 


\section{Acknowedgements}

Communications from Professors H. D. Zeh, E. Joos, and W. H. Zurek have contributed significantly to the form and content of this paper.

\section{References}

[1] J. Barrett. Everett's Relative-State formulation of Quantum Mechanics Stanford Encyclopedia of Philosophy, http://plato.stanford.edu/entries/qm-everett

[2] D.Deutsch. Quantum Theory as a Universal Physical Theory, Int. J. Theor. Phys. 24, 1-41 (1985)

[3] S. Foster and H. Brown. On a Recent Attempt to Define the Interpretation Basis in the Many Worlds Interpretation of Quantum Mechanics, Int. J. Th. Phys 27, 1507-1531 (1988).

[4] E. Joos and H.D. Zeh. The Emergence of Classical Properties Through Interaction With the Environment, Z. Phys. B59, 223-243 (1985); D. Guilini, E. Joos, C. Kiefer, J. Kupsch, I.O. Stamatiscu, and H.D. Zeh, Decoherence and the Appearance of a Classical World in Quantum Theory, Springer-Verlag, Berlin, New York, 1996.

[5] John Klauder and Bo-Sture Skagerstam, Coherent States, World Scientific, Singapore, 1985, p.12.

[6] D.Deutsch. The Structure of the Multiverse, http://www.arXiv.org/abs/quant-ph/0104033

[7] R.P. Feynman. Quantum Mechanical Computers.

Optics News 11, 11-20 (1985). 
[8] W. Zurek. Decoherence, Einselection, and the Existential Interpretation (the rough guide). Phil. Trans. R. Soc. Lond. A 356, 1793-1821 (1998).

[9] H. P. Stapp, in Symposium on the Foundations of Modern Physics 1990, eds. P. Lahti and P. Mittelstaedt, World Scientific, Singapore, 1991. Sec. 3. 